\title{
Ventrolateral prefrontal cortex updates chosen value according to choice set size
}

Fujiwara, Juri ; Usui, Nobuo ; Eifuku, Satoshi ; Iijima, Toshio ; Taira, Masato ; Tsutsui, Ken-Ichiro ; Tobler, Philippe N

\begin{abstract}
Having chosen an item typically increases the subjective value of the chosen item, and people generally enjoy making choices from larger choice sets. However, having too many items to choose from can reduce the value of chosen items - for example, because of conflict or choice difficulty. In this study, we investigated the effects of choice set size on behavioral and neural value updating (revaluation) of the chosen item. In the scanner, participants selected items from choice sets of various sizes (one, two, four, or eight items). After they chose an item, participants rerated the chosen item, and we quantified revaluation by taking the difference of postchoice minus prechoice ratings. Revaluation of chosen items increased up to choice sets of four alternatives but then decreased again for items chosen from choice sets of eight alternatives, revealing both a linear and a quadratic effect of choice set size. At the time of postchoice rating, activation of the ventrolateral pFC (VLPFC) reflected the influence of choice set size on parametric revaluation, without significant relation to either prechoice or postchoice ratings tested separately. Additional analyses revealed relations of choice set size to anterior cingulate and insula activity during actual choice and increased coupling of both regions to revaluation-related VLPFC during postchoice rating. These data suggest that the VLPFC plays a central role in a network that relates choice set size to updating the value of chosen items and integrates choice overload with value-enhancing effects of larger choice sets.
\end{abstract}

DOI: https://doi.org/10.1162/jocn_a_01207

Posted at the Zurich Open Repository and Archive, University of Zurich

ZORA URL: https://doi.org/10.5167/uzh-146513

Journal Article

Published Version

Originally published at:

Fujiwara, Juri; Usui, Nobuo; Eifuku, Satoshi; Iijima, Toshio; Taira, Masato; Tsutsui, Ken-Ichiro; Tobler, Philippe N (2018). Ventrolateral prefrontal cortex updates chosen value according to choice set size. Journal of Cognitive Neuroscience, 30(3):307-318.

DOI: https://doi.org/10.1162/jocn_a_01207 


\title{
Ventrolateral Prefrontal Cortex Updates Chosen Value According to Choice Set Size
}

\author{
Juri Fujiwara $^{1,2,3,4}$, Nobuo Usui ${ }^{4}$, Satoshi Eifuku ${ }^{3}$, Toshio Iijima ${ }^{2}$, Masato Taira ${ }^{4}$, \\ Ken-Ichiro Tsutsui ${ }^{2}$, and Philippe N. Tobler ${ }^{1}$
}

\begin{abstract}
Having chosen an item typically increases the subjective value of the chosen item, and people generally enjoy making choices from larger choice sets. However, having too many items to choose from can reduce the value of chosen itemsfor example, because of conflict or choice difficulty. In this study, we investigated the effects of choice set size on behavioral and neural value updating (revaluation) of the chosen item. In the scanner, participants selected items from choice sets of various sizes (one, two, four, or eight items). After they chose an item, participants rerated the chosen item, and we quantified revaluation by taking the difference of postchoice minus prechoice ratings. Revaluation of chosen items increased up to choice sets of four alternatives but then decreased again
\end{abstract}

\section{INTRODUCTION}

When individuals choose an item, they tend to value that item more highly after than before having chosen it (Chen \& Risen, 2010; Izuma et al., 2010; Sharot, Shiner, \& Dolan, 2010; Sharot, De Martino, \& Dolan, 2009; Brehm, 1956). It is generally assumed that this increased appreciation is due to the very act of choosing. This assumption is tenable because choice itself is valued (e.g., Fujiwara et al., 2013; Leotti \& Delgado, 2011; Jones $\&$ Sugden, 1982) and because an increase in value of the chosen item may prevent inner conflict due to one's positive evaluation of the rejected items (Festinger, 1957). However, as choice set size increases, choice-induced enhancements of value may be limited, for example, due to the increased difficulty of making a decision when faced with many options (Simon, 1995). In line with the notion that consumers may suffer from too many choices (choice overload), large choice sets can lead to reduced motivation to make a choice and decreased satisfaction with choices once they have been made (Johnson et al., 2012; Reutskaja \& Hogarth, 2009; Iyengar \& Lepper, 2000). However, a meta-analysis suggests that, at least in the psychological literature, the choice overload effect

${ }^{1}$ University of Zurich, ${ }^{2}$ Tohoku University, ${ }^{3}$ Fukushima Medical University, ${ }^{4}$ Tokyo Medical and Dental University for items chosen from choice sets of eight alternatives, revealing both a linear and a quadratic effect of choice set size. At the time of postchoice rating, activation of the ventrolateral $\mathrm{pFC}$ (VLPFC) reflected the influence of choice set size on parametric revaluation, without significant relation to either prechoice or postchoice ratings tested separately. Additional analyses revealed relations of choice set size to anterior cingulate and insula activity during actual choice and increased coupling of both regions to revaluation-related VLPFC during postchoice rating. These data suggest that the VLPFC plays a central role in a network that relates choice set size to updating the value of chosen items and integrates choice overload with valueenhancing effects of larger choice sets.

may have been overrepresented and the beneficial effect of larger choice sets may have been underrepresented (Scheibehenne, Greifeneder, \& Todd, 2009). In this study, we investigate the effects of choice set size on choiceinduced value change by varying the number of items to choose from in a stylized shopping task.

Changes in the value of a chosen option appear to be represented in a variety of brain regions, such as the ventrolateral pFC (VLPFC; Jarcho, Berkman, \& Lieberman, 2011; Ochsner \& Gross, 2005) and the striatum (Izuma et al., 2010; Sharot et al., 2009). If the choice process itself were indeed driving the increase in the value of chosen items, then we would expect items that have been chosen from a larger choice set to be valued more highly and affect revaluation regions more strongly than items chosen from a smaller choice set. However, these value-enhancing effects of choice set size may be counteracted by choice overload because of higher cognitive conflict associated with larger choice sets. Such conflict may be exacerbated by having to reject more alternatives during choice from large choice sets. Accordingly, brain regions related to cognitive conflict and negative affect such as the ACC and insula (Jensen et al., 2016; Shenhav, Straccia, Cohen, \& Botvinick, 2014; Fujiwara, Tobler, Taira, Iijima, \& Tsutsui, 2009b) should activate more strongly with larger choice sets, possibly at different time points within the task. 


\section{METHODS}

\section{Participants}

Eighteen right-handed adults (13 men and 5 women, aged $19-27$ years, mean $=22$ years) with no history of neurological, psychiatric, or auditory dysfunction participated in this imaging study. Although a larger sample would have been desirable (e.g., Poldrack et al., 2017), we were limited by available resources. Vision of all participants was normal or corrected to normal. Written informed consent was obtained from each participant. This experiment was conducted in accordance with the Declaration of Helsinki and approved by the ethics committee of the Nihon University School of Medicine.

\section{Task}

We investigated whether the degree of change in the value of chosen items (revaluation) is influenced by choice set size. One to two days before coming to the laboratory, participants provided a preference rating for 1,000 everyday items on a scale of $1=$ low to $9=$ high (Figure 1A). Pictures of items (Figure 1B) were obtained from an online 100-yen shop (similar to an online dollar store). By using only items from this type of popular shop, we not only held the objective item value constant but also ensured that participants were familiar with most items. We adjusted each picture so that they all covered a similar portion of the screen. Each picture was displayed on a white square (5.5- $\mathrm{cm}$ side length, which in the scanner corresponded to $14.3^{\circ}$ visual angle) over a larger black background with the name of the pictured item displayed below the picture inside the white square and the rating scale presented below the white square. The participants were informed that the objective market value of each item was 100 yen (about $\$ 1$ ). On the basis of their individual ratings, we selected the 450 lowest-rated and 450 highest-rated items for each participant for use in the main experiment. We thereby made sure that we had enough items to avoid presenting any item more than once during the main experiment.

In the scanner, participants made two choices and provided one preference rating per trial (Figure 1C), with the second choice and the rating phase being the events of interest for this study. Choice 1 (presented for up to $4 \mathrm{sec})$ was between a monetary amount (10, 30, 50, 70, 90 , or 110 yen) and a number (1, 2, 4, or 8$)$. The number represented the number of low- or high-value items from which participants would choose in Choice 2, if they were to choose the choice (as opposed to the monetary amount) option in Choice 1. Whether the items would be of low or high value was randomly determined from trial to trial and indicated to participants by the color (green or orange, counterbalanced across participants) of the square on which the choice option was presented in Choice 1. Participants were told that, of the 1,000 items they had rated previously, green or orange would represent the 450 items with the highest or lowest rating, re- spectively. The low- and high-value item conditions served to identify subjective value regions at Choice 1 in our previous study (Fujiwara et al., 2013) but were pooled here for all analyses. The monetary amount offered in Choice 1 was determined independently of the properties of any items that might be shown in Choice 2 (value or number of items). In Choice 1, participants essentially traded off choice set size against monetary amount. With everything else being equal, participants chose the monetary option more frequently with increasing monetary amount and the choice option more frequently with increasing set size (Fujiwara et al., 2013). The participants conveyed all their choices verbally through a noise canceling MRI microphone (optiMRI, FOMRI-III; Optoacoustics Ltd., Moshav Mazor, Israel). The chosen option was then highlighted with a red frame for $1 \mathrm{sec}$ by the experimenter, followed by a variable delay (until 5-6 sec had elapsed), during which the screen was blank.

If a participant chose the choice option in Choice 1, the corresponding number of everyday items was displayed in Choice 2; if a participant chose the monetary amount option in Choice 1, the corresponding amount of money was displayed in Choice $2(5 \mathrm{sec})$. To control for overall visual stimulation of Choice 2, we always showed eight squares and repeated items or monetary amounts, respectively. This meant that the proportion of duplicates shown in Choice 2 was inversely related to the number of choice options offered in Choice 1 , resulting in a constant eight-item pictures (e.g., with four options, each item would be shown twice; with two options, each item would be shown four times). After participants made Choice 2, there was a delay of 2-3 sec. Then, the chosen item or monetary amount was presented for $2 \mathrm{sec}$, and participants provided a preference rating for the chosen item, again by indicating a number verbally (postchoice rating phase, $3 \mathrm{sec}$ ). Importantly, the difference between this second rating and the prescanning rating served to quantify revaluation. Finally, there was an intertrial interval of 2-3 sec before the next trial started. All verbal responses were recorded and used to determine RTs (from the onset of each stimulus to the onset of a verbal sound). Here, we analyzed the influence of choice set size on the postchoice rating phase and Choice 2, whereas in our previous article (Fujiwara et al., 2013), we analyzed Choice 1 . Thus, the same data set was used in the prior publication, but a different time point was analyzed, and the analysis focused on a different question (whether choice has value in itself rather than how choice set size affects the updating of value after having chosen).

In total, the participants completed 240 trials $(5$ trials each of 6 monetary amounts $\times 4$ choice set sizes $\times 2$ (low/high) value conditions: $82 \mathrm{~min}$ ) in four sessions. Each session started with a blank screen $(6 \mathrm{sec})$, followed by a text that was to be read $(20 \mathrm{sec})$ and a ready phase $(30 \mathrm{sec})$. In the text reading phase, participants read out the numbers 1-9 to allow for speaker volume adjustment in the scanner control room. Trials in which participants 


\section{A}

Before

scanning: rating (pre)

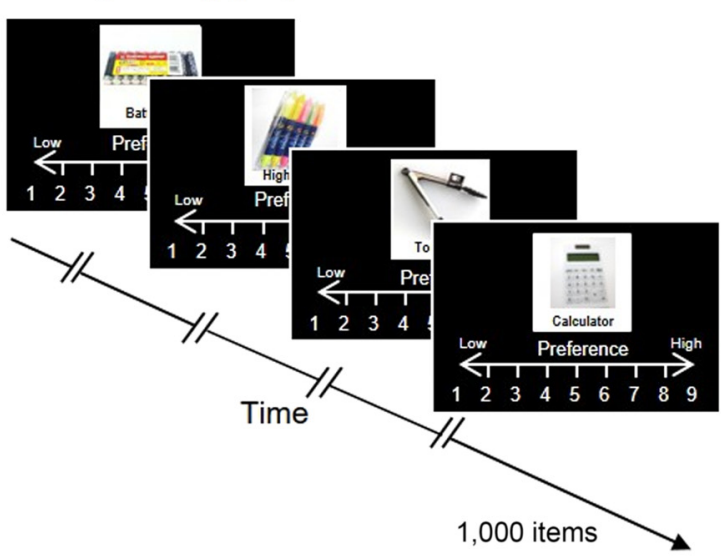

B

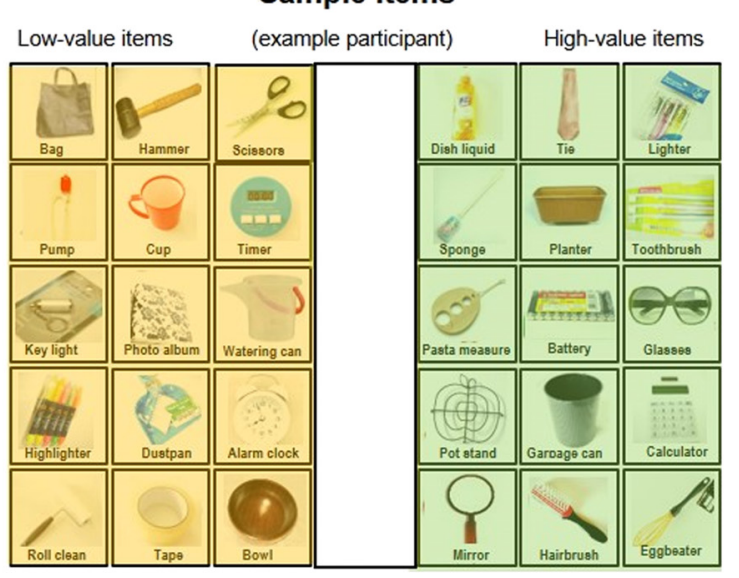

C

During scanning

hoice 1

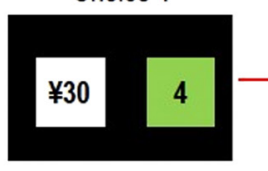

Choice option chosen

\begin{tabular}{|l|l}
\hline & Delay1 \\
\hline
\end{tabular}

Choice2

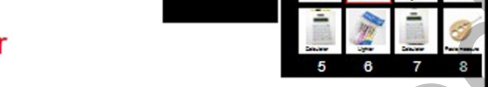

Monetary amount option chosen

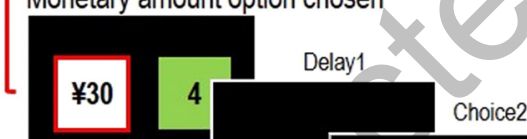

Choice2

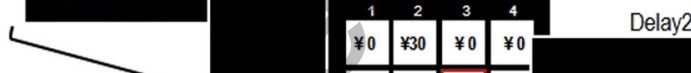

Delay2
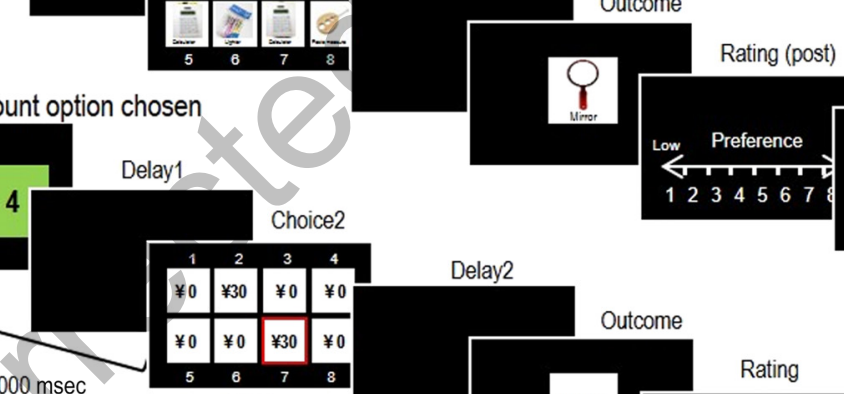

5000 or $6000 \mathrm{msec}$

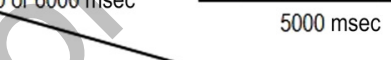

$¥ 10 \quad ¥ 30 \quad ¥ 50 \quad ¥ 70 \quad ¥ 90 \quad ¥ 110$ Monetary amount 2000 or

$2000 \mathrm{msec} 1234567$

Number of high-value choice options

$3000 \mathrm{msec}$

Number of low-value choice options

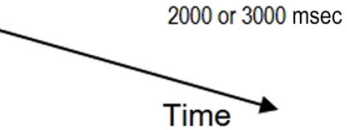

D

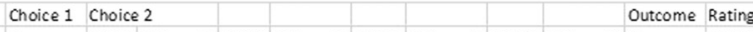

GLM 1 Regressor ONS1 PM reval 1 ONS 2 PM reval 2 ONS 4 PM reval 4 ONS 8 PM reval 8 Regressor ONS 1 PM reval $1 \quad$ ONS 2 PM reval $2 \quad$ ONS 4 PM reval 4 ONS 8 PM reval 8

GLM 2 of no ONS1 PM reval 1ONS 2 PM reval2 ONS 4 PM reval 4 ONS 8 PM reval 8 of no ONS1 PM pre 1 PM post 1 ONS 2 PM pre 2 PM post 2 ONS 4 PM pre 4 PM post 4 ONS 8 PM pre 8 PM post 8 interest

Figure 1. Experimental design. (A) Prechoice rating. One or two days before coming to the laboratory, participants rated their preferences for 1,000 everyday items on a scale of $1=l o w$ to $9=h i g h$. On the basis of the individual ratings, we selected 450 low-value and 450 high-value items for each participant, which were used in the low- and high-value conditions, respectively (see C). (B) Sample items, as classified for one of the participants. All of the items had the same objective market value (100 yen). In the actual experiment, the Japanese, rather than English, word for each item was displayed. (C) Sequential choice task used during scanning. In each trial, participants made two choices and one rating (see Methods for details). Participants had 3 sec to provide the postchoice preference rating for the chosen item. The brain activations reported here are from this phase, with the exceptions of Figure $3 \mathrm{~F}$ and 4, which are from Choice 2. The difference between the rating provided at this stage and rating (pre) was the parameter of primary interest of brain analysis. (D) Experimental regressors of GLMs used to analyze data. GLM 1 served to analyze revaluation-related brain activation during the postchoice rating phase and Choice 2; GLM 2 served to analyze the components of revaluation. In addition to the regressors shown, both GLMs included motion parameters (six regressors per session) and one session regressor. GLM = general linear model; ONS = onset regressor; $\mathrm{PM}=$ parametric modulator; reval $=$ revaluation; $¥=$ yen. The numbers $(1,2,4$, and 8$)$ refer to choice set size. 
did not give a response (mean $=4$ trials per participant) were excluded from further analysis. Thus, the reported results are from completed trials only. On average, participants received earnings of 11,679 yen (about $\$ 130$ ) and 42 items, all of which were delivered to participants approximately 3 months after the experiment. Participants reported that they did not try to add up their earnings during the experiment.

\section{Behavioral Analysis}

We analyzed revaluation as postchoice minus prechoice rating for each chosen item. In a series of linear and quadratic regression analyses, we related individual revaluation levels to (the binary logarithm of) choice set size, using two-tailed tests to test whether parameter estimates differed significantly from zero. Linear regressions capture the revaluation enhancing effect of choice set size, whereas quadratic regressions capture potential choice overload effects on revaluation. As it could be argued that the minimal choice set size of one (no choice) differs from the other choice set sizes (choice), we performed these analyses also separately for choice set sizes larger than one. To assess individual differences, we used the linear individual parameter estimates also for correlations with neural contrasts assessing gradual choice set size effects $(8>4>2>1)$.

\section{fMRI Acquisition and Preprocessing}

All images were acquired using a 1.5-T Siemens Symphony MRI scanner (Siemens, Erlangen, Germany) at Nihon University. Gradient-recalled EPI was used for the fMRI sequence to obtain BOLD contrast functional images. Two thousand four hundred sixty functional scans per participant were acquired using a gradient-echo EPI sequence (20 axial slices in the AC-PC plane; repetition time $=2000 \mathrm{msec}$, echo time $=50 \mathrm{msec}$, flip angle $=90^{\circ}$, field of view $=$ $192 \mathrm{~mm}, 64 \times 64$ matrix, voxel size $=3 \times 3 \times 5 \mathrm{~mm}$, slice gap $=1 \mathrm{~mm}$ ). A T1 anatomical scan of each participant was obtained (192 sagittal slices; repetition time $=2000 \mathrm{msec}$, echo time $=3.93 \mathrm{msec}$, flip angle $=15^{\circ}$, field of view $=256 \times$ $224 \mathrm{~mm}$, in-plane resolution $=1 \times 1 \mathrm{~mm}$, slice thickness $=$ $1 \mathrm{~mm})$.

Preprocessing and data analysis were performed in SPM 8, using Version 17 of the SPM CONN toolbox (www.nitrc.org/ projects/conn, RRID:SCR_009550; Whitfield-Gabrieli \& Nieto-Castanon, 2012) and Version 13 of the SPM permutation toolbox (warwick.ac.uk/snpm). The first 28 functional scans (volume test, noise cancelation, and ready phases) of each session were discarded, which allowed for magnetic saturation. CONN served to detect outliers (global signal $z$ value threshold $=5$ SDs, subject-motion threshold $=0.9 \mathrm{~mm}$ ) and determine global gray matter signal. Functional images were realigned, unwarped, and slice-time corrected. We checked for motion artifacts using the ART-based scrubbing method (Power, Barnes, Snyder,
Schlaggar, \& Petersen, 2012). Gray matter, white matter, and cerebrospinal fluid were segmented, and the functional data were normalized to the Montreal Neurological Institute template. Finally, the data were spatially smoothed with a Gaussian kernel set at $8 \mathrm{~mm}$ FWHM.

\section{fMRI Data Analysis}

The functional data were submitted to two event-related general linear model (GLM) analyses (Figure 1D). GLM 1 assessed revaluation at the postchoice rating phase and at Choice 2, whereas GLM 2 considered the constituents of revaluation at the postchoice rating phase. Regressors were formed by convolving stimulus functions with a canonical hemodynamic response function. Participantspecific motion parameters were modeled as covariates of no interest. A high-pass filter with a cutoff period of $128 \mathrm{sec}$ was used to remove low-frequency drifts.

We expected the neural effects of revaluation to be strongest during the postchoice rating phase because, at this time point, the previous value of the item would have to be updated with its new value, after having been chosen from choice sets of variable sizes. In both GLMs, activation was thus modeled from the onset of the rating screen until rating was completed (variable duration, average $=2.0 \mathrm{sec}$ ), using four regressors according to choice set size in Choice 2 (one, two, four, and eight items). In GLM 1, we used the degree of revaluation as parametric modulators for each regressor at postchoice rating. This allowed us to detect linear activation changes related to value increase and compare them between different choice set sizes $(8>4>2>1)$. We also included regressors for Choice 1 (modeled with a duration corresponding to the trial-specific RTs) and Outcome in the model as events of no interest because they potentially explained some of the variance in brain activation. Moreover, to assess temporal specificity of the revaluation findings, we modeled choice-set-size-dependent revaluation also at Choice 2 (including one onset regressor and one parametric modulator for each choice set size). For Choice 2, we considered set size effects also for the onset regressors rather than parametric revaluation modulators. Given that the behavioral data showed evidence for choice overload effects primarily with eight alternatives, we compared this condition against the average of the others $(8-[1 / 3 \times(4+2+1)])$. In total, each session of GLM 1 was modeled with 26 regressors. These consisted of four regressors with one parametric revaluation modulator each at the time of postchoice rating (giving eight regressors), four regressors with one parametric revaluation modulator each at the time of Choice 2 (eight regressors), one regressor each for Choice 1, Outcome, and global gray matter signal. Finally, we also included movement parameters (six regressors) and a nonspecific session regressor.

GLM 2 served to test whether the revaluation-related activations identified with GLM 1 can be explained by 
prechoice or postchoice ratings alone. For GLM 2, we therefore replaced the parametric revaluation modulators of each choice set size with their components, namely, a parametric prechoice rating modulator and a parametric postchoice rating modulator, without orthogonalization. Apart from this change, everything else (durations, regressors of no interest, contrasts) remained the same as in GLM 1. We needed a second GLM because revaluation is a linear combination of postchoice and prechoice rating and a GLM containing all three parametric modulators would not have been estimable because of perfect collinearity.

We performed a whole-brain psychophysiological interaction (PPI) analysis (Friston et al., 1997) to determine whether choice-overload-like processes during Choice 2 affected revaluation-related regions in VLPFC (where choice set size affected revaluation-related activity). We constructed a GLM that included the following regressors: (1) one physiological regressor (time course of activity extracted from seed regions in the bilateral anterior insula identified with the choice overload contrast), (2) four psychological regressors (one for each choice set size), and (3) four PPI regressors (multiplications of 1 and 2).

Because of the relatively small sample size $(n=18)$, we applied nonparametric tests for statistical inference (Nichols \& Holmes, 2002). Specifically, we used Version 13 of the SPM permutation toolbox (www.sph.umich. edu/ni-stat/SnPM/) to permute the labels of the four choice set sizes (i.e., 1, 2, 4, and 8) and assess whether the actual differences between choice set sizes were significant based on the resulting pseudo $t$ statistic. All results shown in the figures survive whole-brain correction at the peak level $(p<.05)$. Uncorrected results are reported in Tables 1 and 2 as well as on neurovault. org (neurovault.org/collections/2886/, Figure 3A; neurovault.org/collections/2872/, Figure 4A; neurovault.org/ collections/2895/, Figure 5A). Reported voxels conform to Montreal Neurological Institute coordinate space, with the right side of the image corresponding to the right side of the brain.

Table 1. Stronger Parametric Revaluation Coding for Items Chosen from Larger Choice Sets during the Postchoice Rating Phase

\begin{tabular}{lcrrrrr}
\hline & & $x$ & $y$ & $z$ & Z Score & Voxels \\
\hline VLPFC & $\mathrm{L}$ & -46 & 30 & -12 & 5.04 & 143 \\
Caudate & $\mathrm{R}$ & 4 & 2 & -4 & 3.83 & 20 \\
Middle frontal gyrus & $\mathrm{R}$ & 34 & 28 & 44 & 3.60 & 24 \\
Medial orbitofrontal gyrus & $\mathrm{R}$ & 12 & 54 & -8 & 3.56 & 11 \\
Rectal gyrus & $\mathrm{L}$ & -4 & 36 & -24 & 3.55 & 25 \\
Posterior cingulate gyrus & $\mathrm{R}$ & 4 & -52 & 34 & 3.39 & 11 \\
Supramarginal gyrus & $\mathrm{R}$ & 48 & -50 & 34 & 3.28 & 16 \\
\hline
\end{tabular}

$p<.001$, uncorrected, $>10$ voxels.
Table 2. Preferential Effects of Largest Choice Set (8) as Compared with Smaller Choice Sets (1, 2, and 4) at Choice 2

\begin{tabular}{lrrrrrr}
\hline & & $x$ & $y$ & $z$ & $Z$ Score & Voxels \\
\hline Insula & $\mathrm{L}$ & -28 & 22 & 6 & 4.69 & 342 \\
& $\mathrm{R}$ & 34 & 22 & 10 & 5.95 & 423 \\
Anterior cingulate gyrus & $\mathrm{R}$ & 12 & 22 & 24 & 4.16 & 114 \\
Posterior cingulate gyrus & $\mathrm{R}$ & 6 & -66 & 10 & 4.59 & 1,443 \\
Superior frontal gyrus & $\mathrm{R}$ & 12 & 2 & 72 & 3.59 & 32 \\
Medial frontal gyrus & $\mathrm{R}$ & 8 & 6 & 54 & 3.52 & 60 \\
Lingual gyrus & $\mathrm{L}$ & -20 & -94 & -8 & 4.70 & 318 \\
\hline
\end{tabular}

$p<.001$, uncorrected, $>10$ voxels.

\section{RESULTS}

\section{Behavioral Data}

We used behavioral methods and fMRI to investigate how the act of choosing from different choice set sizes changes the subsequent valuation of chosen items. Before the experiment, participants provided a preference rating for 1,000 everyday items of the same objective value, 100 yen (Figure 1A). These ratings served as the basis for participant-specific classification into 450 lowest-rated and 450 highest-rated items (Figure 1B). During the experiment, participants rated items for a second time after having chosen them from differently sized choice sets (Figure 1C). As would be expected, participants chose the choice option in Choice 1 more frequently with larger choice sets and a higher initial value. Indeed, at Choice 1 , the subjective value of the choice option increased monotonically with choice set size, such that adding one additional choice alternative on average increased the value of the choice option by 3.6 yen (see Fujiwara et al., 2013, also for neural data at Choice 1). The subjective value of larger choice sets increased also with choice sets larger than one alternative. For example, the subjective value of choice sets of eight alternatives was about 20 yen higher than the subjective value of choice sets of two alternatives $(t(17)=3.62, p<.005$, two-tailed). Thus, larger choice sets monotonically increased the value of choice.

Next, we asked how choice set size affected revaluation measured at the postchoice rating phase by assessing how much the ratings changed after the item had been chosen. Specifically, we compared the average post-pre ratings given to items that participants had chosen in Choice 2. Qualitatively, revaluation increased up to choice sets of four alternatives but then decreased again for items chosen from choice sets of eight alternatives (Figure 2A), indicating that choice set size affected revaluation differently from Choice 1. Statistically, regression analyses confirmed that the revaluation rating data could be captured by the combination of a linear effect and a quadratic effect of choice set size. Both effects were significant when considering all choice set sizes (linear: $t(17)=$ 
Figure 2. Behavior. (A) Average amounts of revaluation (calculated as postchoice minus prechoice rating) of the chosen item as a function of choice set size. (B) Average RT in Choice 2 as a function of choice set size. Error bars represent SEM.
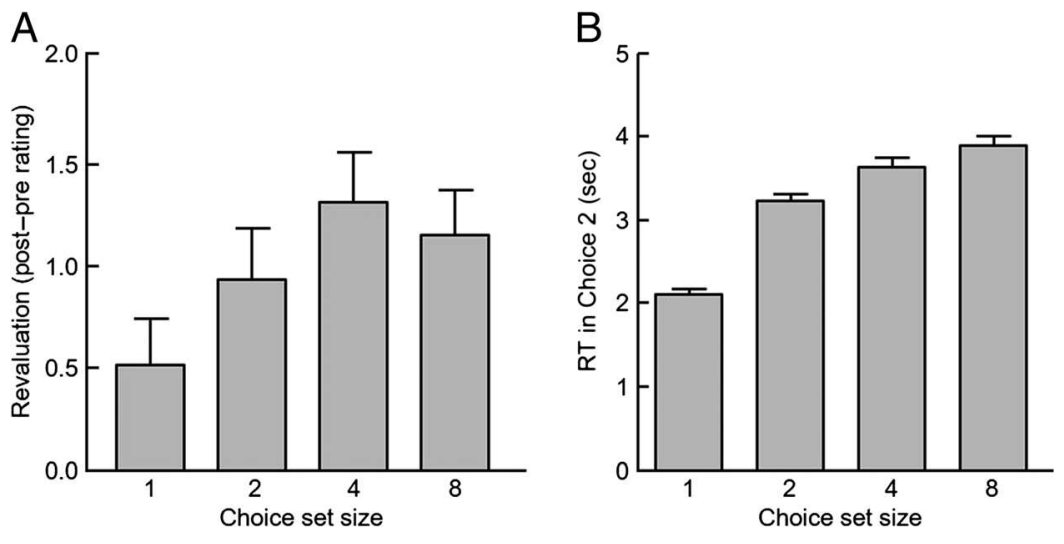

$3.96, p<.001$; quadratic: $t(17)=2.43, p<.05$; both twotailed). Although the linear effect reached only trend level significance $(t(17)=1.83, p=.08$, two-tailed) when considering only Choice sets 2,4 , and 8 , it was also not significantly different between Choice sets 1, 2, and 4 and 2, 4, and $8(t(17)=1.81, p=.09$, two-tailed). Moreover, the quadratic effect was significant with Choice sets 2, 4, and 8 only $(t(17)=2.58, p<.05$, two-tailed). Pairwise comparisons showed that Choice set sizes 2 and 4 were significantly different from each other $(t(17)=3.25, p<.005$, two-tailed), whereas Choice set sizes 2 and 8 were not $(t(17)=1.60, p=.13$, two-tailed). These data suggest that increasing choice set size both enhances revaluation (linear effect) and results in a choice-overload-like (quadratic) effect on revaluation. These two aspects may be combined for revaluation, with the quadratic effect possibly reflecting higher cognitive conflict induced by larger choice sets at Choice 2. Such conflict may be exacerbated by having to reject more alternatives during the choice process with large choice sets, particularly during Choice 2.

Next, we tested whether choice difficulty, measured as average RT from the onset of item pictures to the onset of a verbal sound, varied as a function of choice set size in Choice 2. We found that it $\operatorname{did}(F(3,68)=42.3 p<.0001$; Figure 2B). RTs increased with choice set size, also for choice set sizes with more than one alternative (Figure 2B), resulting in significant differences between all choice set sizes (1 vs. $2: 2.28$ vs. $3.22 \mathrm{sec}, t(17)=$ 10.03; 2 vs. $4: 3.22$ vs. $3.53 \mathrm{sec}, t(17)=5.24 ; 4$ vs. 8 : 3.53 vs. $3.80 \mathrm{sec}, t(17)=6.97$; all $p s<.0001$, two-tailed). In contrast, rating times for the postchoice rating phase did not differ as a function of choice set size $(F(3,68)=$ $1.2, p=.3)$. Together, these results suggest that higher difficulty as expressed by longer RTs with larger choice sets affected primarily Choice 2 .

\section{Neuroimaging Data}

\section{Choice Set Size Effects on Revaluation}

We first identified regions in which activation related to revaluation (postchoice minus prechoice rating) was in- fluenced by choice set size. Specifically, at the time of postchoice rating, we tested for stronger parametric revaluation effects as choice set size increased $(1<2<$ $4<8$ options; to account for RTs in the neural data, they entered the model as the duration of the rating time regressor). The analysis revealed significantly stronger parametric effects for items chosen from larger choice sets in the VLPFC $(x / y / z$ coordinates $=-46 / 30 /-12$; Figure 3A; $p<.05$ whole-brain family-wise error [FWE], peak-level corrected; for activations at $p<.001$ uncorrected, Table 1). Similar to the behavioral revaluation effects (Figure 2A), the average parametric revaluation effect in the VLPFC region was strongest at the choice set size of four alternatives and then decreased again (Figure 3B). These data suggest that the behavioral effects of choice set size on revaluation are reflected in VLPFC.

To investigate individual differences, we performed a brain-behavior correlation analysis between neural activity capturing choice set size effects on revaluation $(8>4>2>1)$ and individual beta weights from the behavioral regression. We performed this analysis within the VLPFC cluster identified above. For the linear beta weights, we found a significant correlation (Figure 3C; $r=.48, p=.04)$. The finding shows that the more participants enhanced the value of the chosen item as a function of choice set size, the stronger was the linear effect of choice set size on revaluation in VLPFC.

\section{Control Analyses}

Next, we asked whether the choice set size effects on VLPFC responses are specifically related to revaluation, that is, to updating the previous value with the current value of the chosen item, as opposed to being related to the previous or current value of the chosen item. We therefore used a second GLM in which we replaced the revaluation parametric modulator of the postchoice rating with its two components, namely, a parametric prechoice rating modulator and a parametric postchoice rating modulator. For each of these modulators, we tested the same contrast $(1<2<4<8)$ as in GLM 1 . The VLPFC 
Figure 3. Stronger parametric revaluation coding for items chosen from larger choice sets during the postchoice rating phase. (A) Location in VLPFC with stronger parametric activation for revaluation of items chosen from larger choice sets $(x / y / z=-46 / 30 /-12$, $p<.05$ whole-brain FWE peak corrected). (B) Average effects in the VLPFC region identified in A. The bar plots shown in B illustrate the effects shown in A without further inference. (C) Neural revaluation correlates with behavioral revaluation. Individual beta weights from the linear effect of choice set size in behavioral revaluation correlated with corresponding brain activation in the VLPFC cluster shown in A. (D-F) Absence of significant choice set size effects in the VLPFC region identified in A for parametric previous value (D) and parametric current value (E) at the postchoice rating phase and for parametric revaluation (post-pre rating; F) at Choice 2.
A

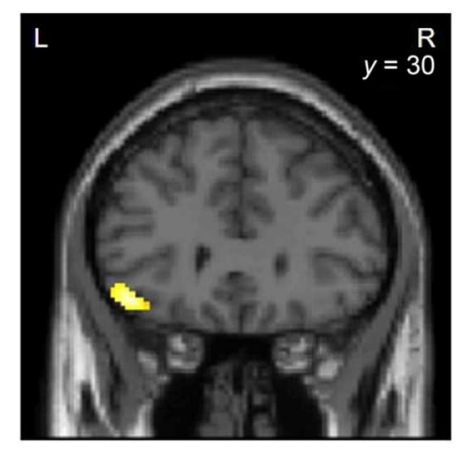

C

Individual VLPFC activation correlates with behavioral revaluation slope

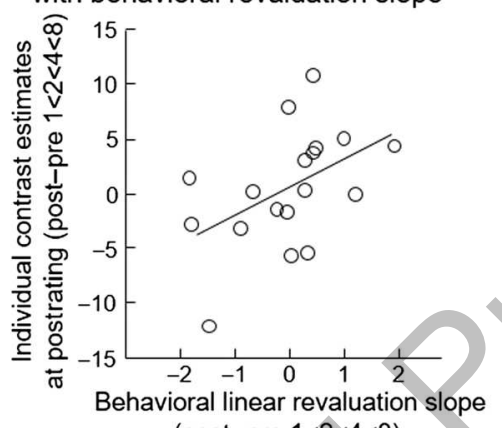
(post-pre $1<2<4<8$ )

\section{E}
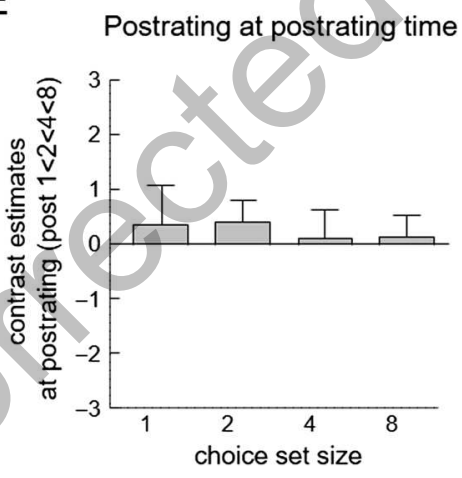

B Revaluation at postrating time

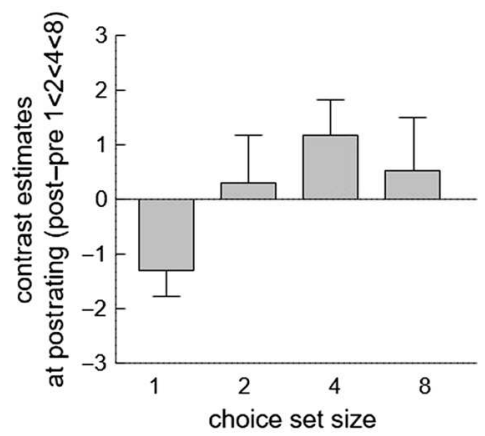

D Prerating at postrating time

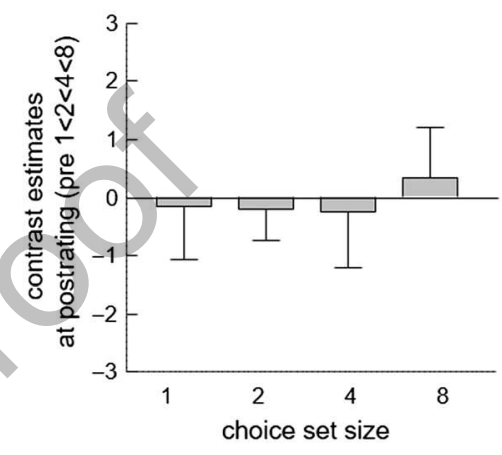

$\mathrm{F}$ Revaluation at Choice 2

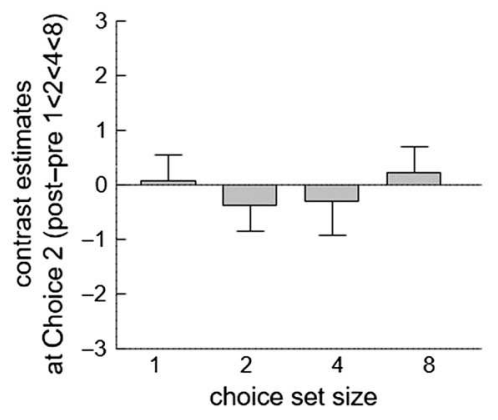

region where choice set size affected revaluation-related activation showed no effect of choice set size on either prechoice or postchoice valuation (Figure 3D and E), even at the lower threshold of $p<.05$, uncorrected. Accordingly, choice set size was unrelated to the average parametric effects of prechoice and postchoice ratings in VLPFC. Thus, the effect of choice set size on value coding in the VLPFC concerns primarily revaluation rather than its components of previous and current values.

To test the temporal specificity of choice set size effects on revaluation-related VLPFC activation, we also assessed Choice 2 rather than the postchoice rating phase (GLM 1). We found no significant effect of choice set size on revaluation-related activity in VLPFC even at the lower threshold of $p<.05$, uncorrected. Accordingly, choice set size was unrelated to average revaluation effects in the VLPFC (Figure 3F) at Choice 2. Together, the findings suggest that choice set size affected revaluation primarily at the postchoice rating phase.

\section{Effects of Eight-Alternative Choice Sets on Choice 2}

In the behavioral data (Figure 2A), choice overload effects became apparent only with a choice set size of eight alternatives in our paradigm. We therefore explored whether there was something special about this condition at Choice 2, using GLM 1. Specifically, we compared the activation to choice set size of eight alternatives (onset regressors rather than parametric revaluation modulators) against activation to the mean of smaller choice set sizes (i.e., 1, 2, and 4). We found that activations in the bilateral insula were particularly strong for the largest choice set size $(x / y / z$ coordinates $=-28 / 22 / 6,34 / 22 / 10$; Figure 4; $p<.05$ whole-brain FWE, peak-level corrected; 
Figure 4. Preferential effects of the largest choice set (8) as compared with smaller choice sets $(1,2$, and 4$)$ at Choice 2 . (A) Location in the bilateral insula with significantly stronger activation for the largest choice set compared with the average of the three smaller choice sets. (B) Average effects in the left insula region $(x / y / z=-28 / 22 / 6$; $p<.05$ whole-brain FWE, peaklevel corrected) identified in A. The bar plots shown in $\mathrm{B}$ illustrate the effects shown in A without further inference. (C, D) Correlation in the left and right insula between individual differences in revaluation for the largest choice set (8) compared with smaller choice sets $(1,2$, and 4$)$ and the corresponding activity elicited by the contrast $(8-(1,2$, and 4)) at Choice 2. Insula peaks identified in A were correlated with individual behavioral effects $(r>.6, p<.01)$.

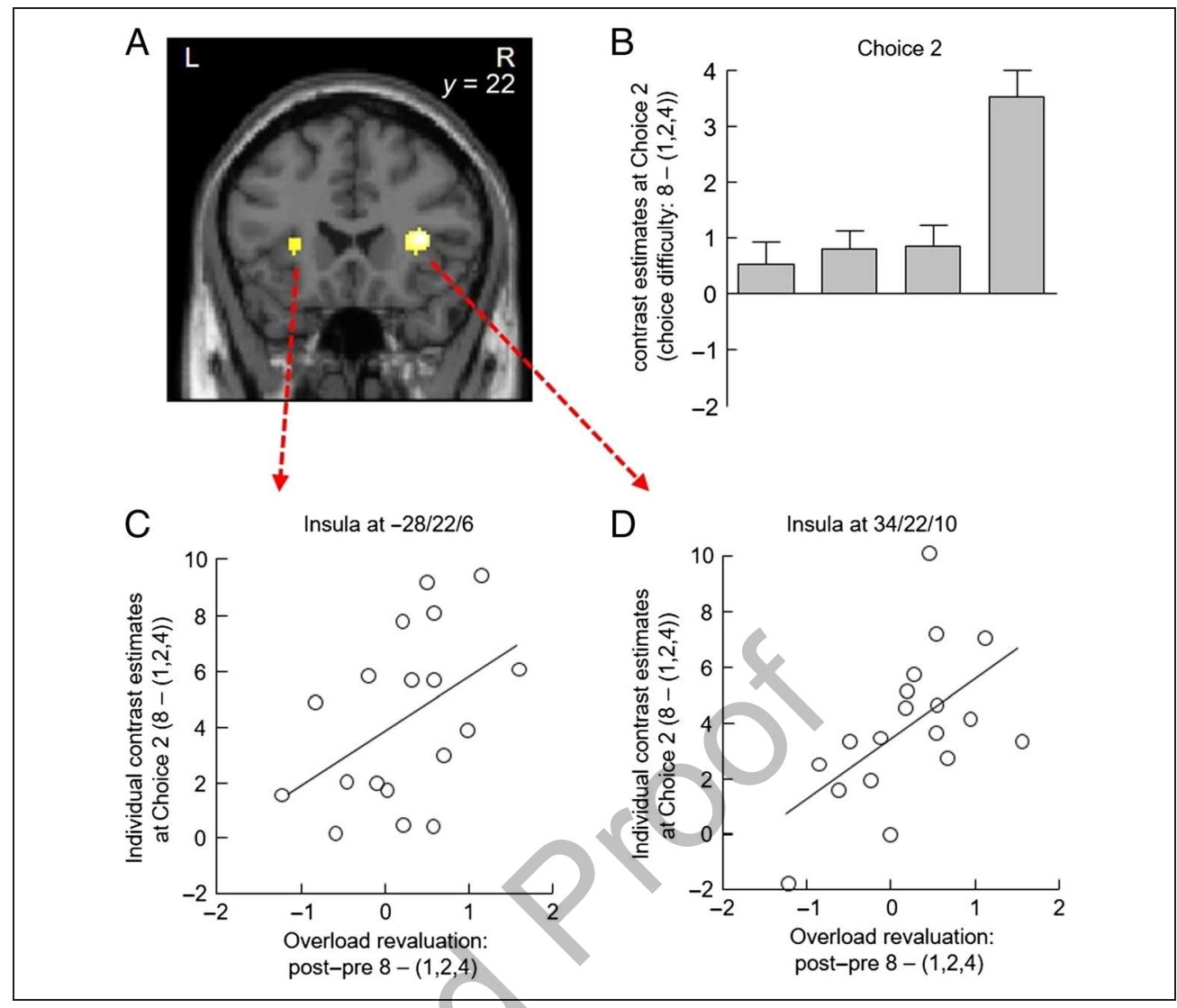

Table 2). These data suggest that the insula at Choice 2 may contribute to choice overload, arising, for example, from enhanced choice difficulty or negative affect from rejecting alternatives in the largest choice set.

To test whether and how these findings relate to revaluation, we correlated activity at the bilateral insula peaks with behavioral revaluation effects particularly of eight choice alternatives. Thus, we extracted the peak activations from Figure $4 \mathrm{~A}$ and correlated them with individual differences in revaluation effects for items chosen from choice sets with eight alternatives as opposed to items chosen from smaller choice sets (i.e., 1, 2, and 4). We found that the bilateral insula peaks showed a significant relation to revaluation (Figure $4 \mathrm{C}$ and $\mathrm{D}$ ). Thus, insula activation specific to the largest choice set during Choice 2 may be particularly sensitive to individual differences in overload-like revaluation effects.

\section{Functional Connectivity between Choice Overload and Revaluation}

Next, we asked whether and how the insular regions that showed particularly strong activation with eight alternatives at Choice 2 would communicate with the revaluation-related region in VLPFC. To answer this question, we performed a PPI analysis to investigate choice-set-size-dependent functional connectivity of the insula regions showing choice-overload-like effects at Choice 2 and the VLPFC region related to revaluation at the postchoice rating phase. We created 6-mm sphere VOIs from peak coordinates (Figure 4A: - 28/22/6, 34/22/ 10) identified at Choice 2 and used them as seed regions at the time of revaluation. The PPI analysis revealed stronger coupling of the left insula with VLPFC for eight alternatives than for smaller choice set sizes (Figure 5A; left insula-VLPFC: $-32 / 30 /-14, p<.05$ whole-brain FWE, peak-level corrected). These VLPFC regions overlapped (Figure 5B) with that showing choice set size effects on revaluation-related activity identified above (Figure 3A). These findings suggest that cross talk between regions showing choice overload effects at Choice 2 and VLPFC regions involved in revaluation was particularly intimate for the largest choice set.

\section{DISCUSSION}

In this study, we investigated the behavioral and neural mechanisms underlying the influence of choice set size on revaluation. It is well known that the value of chosen items can increase (Chen \& Risen, 2010; Izuma et al., 2010; Sharot et al., 2009, 2010; Brehm, 1956). The fact that this holds also for relatively low-value items like the 100-yen items studied here adds to the generality of this finding. Importantly, we found that choice set size effects on revaluation were reflected in the VLPFC (Figure 3), with a relation to individual enhancement of 


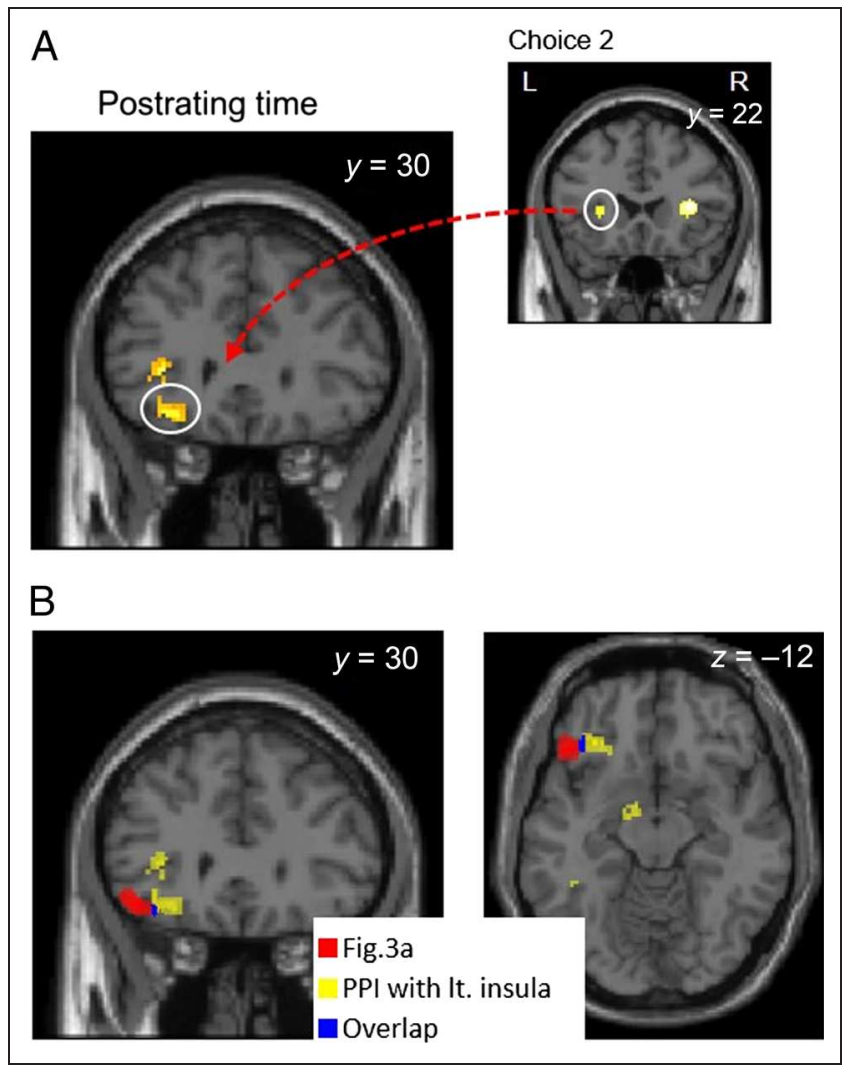

Figure 5. Functional connectivity between choice overload and revaluation regions at the postchoice rating phase. (A) Locations in the VLPFC showing stronger connectivity with the left (lt.) insula for revaluation of items chosen from the largest choice set as compared with all other choice sets $(-32 / 30 /-14 ; p<.05$ whole-brain FWE, peak-level corrected). (B) Regions identified with the PPI analysis overlapped with the region shown in Figure 3A (note that the extent of the overlap is threshold dependent).

revaluation by choice set size. These findings suggest that value updating processes of the VLPFC are sensitive to choice set size. Moreover, effects reminiscent of choice overload occurred predominantly during choice itself in the insula, which also showed choice-set-size-dependent coupling with VLPFC in the postchoice rating phase.

\section{Linear and Quadratic Effects of Choice Set Size on Behavioral Revaluation}

With an increasing choice set size, revaluation of chosen items increased and then decreased again, which contrasts with the monotonic impact of choice set size on the value of choice itself. Thus, in the revaluation stage, a relatively well-established, value-enhancing linear effect of choice set size (Scheibehenne et al., 2009) appears to be combined with a quadratic overload-like effect, which may be due to choice difficulty, conflict, or negative affect. With large choice set sizes, one has to compare and reject more alternatives, which may explain why items chosen from large choice sets are valued less than items chosen from intermediate choice sets, as reflected by the quadratic effect surfacing in our revaluation data. Please note that a basic implication of our definition of revaluation is that remembered previous value needs to be integrated and updated with experienced current value and that this process may be influenced by the recent choice process. At least, the quadratic effect may arise from the choice process itself, whereas the linear effect may reflect either the higher likelihood of a better item being included in larger choice sets or the value of choice itself (Fujiwara et al., 2013). In any case, the combined linear and quadratic effects suggest that choice set size impacts revaluation in an intricate and hitherto underappreciated fashion.

\section{Alternative Explanations of Choice Set Size Effects on Behavioral Revaluation}

It is conceivable that familiarity influenced the revaluation of items chosen from different choice sets. However, we believe that it is unlikely that the effects can be explained by familiarity as our participants were all used to buying from 100-yen shops; all the items were common, everyday items; and participants already owned some of the more familiar items and therefore did not choose them in our task. Moreover, items were randomly assigned to different choice set sizes, suggesting that average familiarity was roughly equated across different conditions.

Although revaluation in tasks like ours has traditionally been interpreted as choice induced (Brehm, 1956), Chen and Risen (2010; see also Izuma \& Murayama, 2013) have reminded us more recently that care needs to be taken with this interpretation. They suggested that seemingly choice-induced value enhancements of the chosen item can be explained by noise in subjective value representations (see also Alós-Ferrer \& Shi, 2015, who showed that the argument of Chen and Risen only holds for items with the same prechoice rating). With everything else being equal, items that are chosen are likely to have slightly higher value than items that are not chosen, and the postchoice rating may simply pick up the fact that the prechoice rating was too low. The size of this artifact could in principle increase with choice set size. However, in our behavioral data, the value-enhancing effect of choice set size had a peak at a choice set size of four alternatives and then decreased again. This pattern is in line with the notion of the choice overload literature that having too many choice alternatives can reduce postchoice satisfaction with the chosen item and thereby counteract choice-induced value increases (Johnson et al., 2012; Reutskaja \& Hogarth, 2009; Iyengar \& Lepper, 2000). Although the confound described by Chen and Risen (2010) appears to be at odds with the data, we cannot entirely rule it out, and future research should use their control procedure of reverting the order of the second rating and choice. 


\section{Parametric Revaluation Signals in VLPFC Show Impact of Choice Set Size}

Our data suggest that choice-set-size-dependent revaluation effects are represented in the VLPFC (and, to a lesser degree, classic value regions such as the caudate and the medial OFC). One process in which the VLPFC has been typically involved is emotion regulation. For example, VLPFC activation is elevated when people downregulate their emotional response to attractive others (Meyer, Berkman, Karremans, \& Lieberman, 2011), highly aversive images (Ochsner \& Gross, 2005; Phan et al., 2005), anticipated physical pain (Kalisch et al., 2005), and viewed sad (Levesque et al., 2003) or erotic films (Beauregard, Levesque, \& Bourgouin, 2001). Conversely, emotional upregulation may underpin the parametric relief signals in VLPFC (Fujiwara, Tobler, Taira, Iijima, \& Tsutsui, 2009a) that reflect how much better the actual outcome of the chosen option is compared with that of the nonchosen option. It is conceivable that our participants engaged in similar forms of emotion regulation during postchoice rating. For example, they could have downregulated their emotional response to having had to reject highly rated alternatives and balanced it with the higher value of larger choice sets. Alternatively, they could have attempted to upregulate their emotional response so as to convince themselves that they had made the right choice although they had to reject several other tempting choice items. In any case, our data raise the question of whether emotion regulation mediates the impact of choice set size on the revaluation of chosen items.

The VLPFC, specifically lateral OFC, has been associated with assigning credit to chosen stimuli and with connecting representations of specific stimuli with those of specific rewards (Noonan, Kolling, Walton, \& Rushworth, 2012). Credit assignment may be particularly relevant for larger choice set sizes, when it is more difficult to keep track of the chosen alternative and value updating more important. Our data suggest that the VLPFC may be preferentially involved in adjusting rather than simply assigning credit.

\section{Links to Pathophysiology}

Changes in VLPFC activity and connectivity have been associated with bipolar (Phillips, Ladouceur, \& Drevets, 2008) and major depressive (Rive et al., 2013) disorder. Compared with healthy controls, patients with major depressive disorder show increased VLPFC activity during attentional and cognitive forms of emotion regulation but reduced activity during negative feedback (Rive et al., 2013). Moreover, they also show reduced VLPFC connectivity with the hippocampus and other regions (Frodl et al., 2010). Further impairments of valuation processes in VLPFC have been reported for depressed patients with a suicide attempt compared with depressed patients without a suicide attempt (Olié et al., 2015).
The effects of choice set size on revaluation in these conditions remain to be investigated. To the extent that revaluation draws on emotion regulation and that changes in VLPFC activity reflect impaired emotion regulation, one could hypothesize that the effects of choice set size on revaluation are less pronounced in patients with major depressive disorder.

\section{Choice Overload-like Effects in Insula}

We found that the insula is particularly active for the largest choice set during Choice 2. These activations could reflect choice difficulty or negative affect, which would reduce the value of items chosen from larger choice sets. In line with this interpretation, many studies have found the insula to be involved in processing aversive emotional states, conflict, pain, and distress (Jensen et al., 2016; Shenhav et al., 2014; Corradi-Dell'Acqua, Hofstetter, \& Vuilleumier, 2011; Fujiwara et al., 2009b; Wicker et al., 2003; Calder, Lawrence, \& Young, 2001; Damasio et al., 2000; Reiman et al., 1997).

The insula region showing choice-overload-like effects was more strongly coupled with VLPFC during revaluation of the option chosen from the largest choice set size. Thus, the effects of choice set size during Choice 2 appear to be reflected in choice-set-size-dependent coupling with revaluation regions at the time of revaluation, suggesting one possible mechanism of how negative emotions and choice difficulty could influence revaluation. Together with the insula activations at Choice 2, these findings contextualize our VLPFC data. They are in line with a model of revaluation that involves conflict and negative emotion due to having to reject unchosen items and updating of previous valuation. It should be noted though that this particular model of revaluation corresponds to only one of several possibilities in which value can be updated (rather than overwriting prior with the current value). More generally, our findings are in line with the notion that, in addition to activation, connectivity as studied with PPI can provide valuable and reliable (Smith, Gseir, Speer, \& Delgado, 2016) insights into neurocognitive function.

\section{Relation to Consumer Research}

Finally, we would like to place this study in the larger context of consumer research. In many countries, supermarkets and hypermarkets are popular and highly profitable. There are several potential reasons why the choice they provide is valued, for example, preference matching (consumers are more likely to find their most highly preferred items) and the valuation of choice as such (consumers value having more choices; e.g., Fujiwara et al., 2013; Jones \& Sugden, 1982). However, another aspect of consumer research suggests that too many choice alternatives can reduce postchoice satisfaction with the chosen item, in agreement with choice overload (Johnson et al., 
2012; Reutskaja \& Hogarth, 2009; Iyengar \& Lepper, 2000; but see Scheibehenne et al., 2009). Our study sheds light on these two effects of choice set size and suggests that part of the reason why hypermarkets are successful may also be that consumers come to like items more that they have bought in stores that provide more choice optionsprovided they bought them without having considered and rejected all the alternative choice options.

In conclusion, our data indicate that choice set size is an important factor for the dynamic calculation of chosen value. Moreover, we show that the VLPFC, in interplay with the insula, underpins choice set size effects on value-based choice and revaluation of chosen items.

\section{Acknowledgments}

This research was supported by the Swiss National Science Foundation (PP00P1_128574, PP00P1_150739, and 00014_165884); the Swiss National Centre of Competence in Research in Affective Sciences; a Grant-in-Aid for Scientific Research (KAKENHI) from the Ministry of Education, Culture, Sports, Science, and Technology (MEXT; nos. 17680027 and 19673002); a Grant-in-Aid for Scientific Research on Priority Areas from the MEXT (nos. 17022009, 18020005, and 20019005); an Academic Frontier Project for Private Universities at Nihon University; and a matching fund subsidy from the MEXT. J. F. was supported by a Global Center of Excellence Program at Tohoku University and an Institutional Program Fellowship for Overseas Visits of Young Researchers from the MEXT.

Reprint requests should be sent to Juri Fujiwara, Department of Systems Neuroscience, Fukushima Medical University, Hikarigaoka 1, Fukushima, Japan 960-1295, or via e-mail juri-f@fmu.ac.jp.

\section{REFERENCES}

Alós-Ferrer, C., \& Shi, F. (2015). Choice-induced preference change and the free-choice paradigm: A clarification.

Judgment and Decision Making, 10, 34-49.

Beauregard, M., Levesque, J., \& Bourgouin, P. (2001). Neural correlates of conscious self-regulation of emotion. Journal of Neuroscience, 21, 6993-7000.

Brehm, J. W. (1956). Post-decision changes in the desirability of alternatives. Journal of Abnormal Psychology, 52, 384-389.

Calder, A. J., Lawrence, A. D., \& Young, A. W. (2001). Neuropsychology of fear and loathing. Nature Reviews Neuroscience, 2, 352-363.

Chen, M. K., \& Risen, J. L. (2010). How choice affects and reflects preferences: Revisiting the free-choice paradigm. Journal of Personality and Social Psychology, 99, 573-594.

Corradi-Dell'Acqua, C., Hofstetter, C., \& Vuilleumier, P. (2011). Felt and seen pain evoke the same local patterns of cortical activity in insular and cingulate cortex. Journal of Neuroscience, 31, 17996-18006.

Damasio, A. R., Grabowski, T. J., Bechara, A., Damasio, H., Ponto, L. L., Parvizi, J., et al. (2000). Subcortical and cortical brain activity during the feeling of self-generated emotions. Nature Neuroscience, 3, 1049-1056.

Festinger, L. (1957). A theory of cognitive dissonance. Evanston, IL: Row, Peterson \& Co.

Friston, K. J., Buechel, C., Fink, G. R., Morris, J., Rolls, E., \& Dolan, R. J. (1997). Psychophysiological and modulatory interactions in neuroimaging. Neuroimage, 6, 218-229.
Frodl, T., Bokde, A. L., Scheuerecker, J., Lisiecka, D., Schoepf, V., Hampel, H., et al. (2010). Functional connectivity bias of the orbitofrontal cortex in drug-free patients with major depression. Biological Psychiatry, 67, 161-167.

Fujiwara, J., Tobler, P. N., Taira, M., Iijima, T., \& Tsutsui, K. (2009a). A parametric relief signal in human ventrolateral prefrontal cortex. Neuroimage, 44, 1163-1170.

Fujiwara, J., Tobler, P. N., Taira, M., Iijima, T., \& Tsutsui, K. (2009b). Segregated and integrated coding of reward and punishment in the cingulate cortex. Journal of Neurophysiology, 101, 3284-3293.

Fujiwara, J., Usui, N., Park, S. Q., Williams, T., Iijima, T., Taira, M., et al. (2013). Value of freedom to choose encoded by the human brain. Journal of Neurophysiology, 110, 1915-1929.

Iyengar, S. S., \& Lepper, M. R. (2000). When choice is demotivating: Can one desire too much of a good thing? Journal of Personality and Social Psychology, 79, 995-1006.

Izuma, K., Matsumoto, M., Murayama, K., Samejima, K., Sadato, N., \& Matsumoto, K. (2010). Neural correlates of cognitive dissonance and choice-induced preference change. Proceedings of the National Academy of Sciences, U.S.A., 107, 22014-22019.

Izuma, K., \& Murayama, K. (2013). Choice-induced preference change in the free-choice paradigm: A critical methodological review. Frontiers in Psychology, 4, 41.

Jarcho, J. M., Berkman, E. T., \& Lieberman, M. D. (2011). The neural basis of rationalization: Cognitive dissonance reduction during decision-making. Social Cognitive and Affective Neuroscience, 6, 460-467.

Jensen, K. B., Regenbogen, C., Ohse, M. C., Frasnelli, J., Freiherr, J., \& Lundström, J. N. (2016). Brain activations during pain: A neuroimaging meta-analysis of patients with pain and healthy controls. Pain, 157, 1279-1286.

Johnson, E. J., Shu, S. B., Dellaert, B. G. C., Fox, C., Goldstein, D. G., Häubl, G., et al. (2012). Beyond nudges: Tools of a choice architecture. Marketing Letters, 23, 487-504.

Jones, P., \& Sugden, R. (1982). Evaluating choice. International Review of Law and Economics, 2, 47-65.

Kalisch, R., Wiech, K., Critchley, H. D., Seymour, B., O'Doherty, J. P., Oakley, D. A., et al. (2005). Anxiety reduction through detachment: Subjective, physiological, and neural effects. Journal of Cognitive Neuroscience, 17, 874-883.

Leotti, L. A., \& Delgado, M. R. (2011). The inherent reward of choice. Psychological Science, 22, 1310-1318.

Levesque, J., Eugene, F., Joanette, Y., Paquette, V., Mensour, B., Beaudoin, G., et al. (2003). Neural circuitry underlying voluntary suppression of sadness. Biological Psychiatry, 53, 502-510.

Meyer, M. L., Berkman, E. T., Karremans, J. C., \& Lieberman, M. D. (2011). Incidental regulation of attraction: The neural basis of the derogation of attractive alternatives in romantic relationships. Cognition E Emotion, 25, 490-505.

Nichols, T. E., \& Holmes, A. P. (2002). Nonparametric permutation tests for functional neuroimaging: A primer with examples. Human Brain Mapping, 15, 1-25.

Noonan, M. P., Kolling, N., Walton, M. E., \& Rushworth, M. F. (2012). Re-evaluating the role of the orbitofrontal cortex in reward and reinforcement. European Journal of Neuroscience, 35, 997-1010.

Ochsner, K. N., \& Gross, J. J. (2005). The cognitive control of emotion. Trends in Cognitive Sciences, 9, 242-249.

Olié, E., Ding, Y., Le Bars, E., de Champfleur, N. M., Mura, T., Bonafé, A., et al. (2015). Processing of decision-making and social threat in patients with history of suicidal attempt: A neuroimaging replication study. Psychiatry Research, 234, 369-377.

Phan, K. L., Fitzgerald, D. A., Nathan, P. J., Moore, G. J., Uhde, T. W., \& Tancer, M. E. (2005). Neural substrates for voluntary 
suppression of negative affect: A functional magnetic resonance imaging study. Biological Psychiatry, 57, 210-219.

Phillips, M. L., Ladouceur, C. D., \& Drevets, W. C. (2008). A neural model of voluntary and automatic emotion regulation: Implications for understanding the pathophysiology and neurodevelopment of bipolar disorder. Molecular Psychiatry, 13, 829-857.

Poldrack, R. A., Baker, C. I., Durnez, J., Gorgolewski, K. J., Matthews, P. M., Munafo, M. R., et al. (2017). Scanning the horizon: Towards transparent and reproducible neuroimaging research. Nature Reviews Neuroscience, 18, 115-126.

Power, J. D., Barnes, K. A., Snyder, A. Z., Schlaggar, B. L., \& Petersen, S. E. (2012). Spurious but systematic correlations in functional connectivity MRI networks arise from subject motion. Neuroimage, 59, 2142-2154.

Reiman, E. M., Lane, R. D., Ahern, G. L., Schwartz, G. E., Davidson, R. J., Friston, K. J., et al. (1997). Neuroanatomical correlates of externally and internally generated human emotion. American Journal of Psychiatry, 154, 918-925.

Reutskaja, E., \& Hogarth, R. M. (2009). Satisfaction in choice as a function of the number of alternatives: When "goods satiate" but "bads escalate". Psychology E Marketing, 26, 197-203.

Rive, M. M., van Rooijen, G., Veltman, D. J., Phillips, M. L., Schene, A. H., \& Ruhé, H. G. (2013). Neural correlates of dysfunctional emotion regulation in major depressive disorder. A systematic review of neuroimaging studies. Neuroscience and Biobehavioral Reviews, 37, 2529-2553.

Scheibehenne, B., Greifeneder, R., \& Todd, P. M. (2009). What moderates the too-much-choice effect? Psychology $\mathcal{G}$ Marketing, 26, 229-253.

Sharot, T., De Martino, B., \& Dolan, R. J. (2009). How choice reveals and shapes expected hedonic outcome. Journal of Neuroscience, 29, 3760-3765.

Sharot, T., Shiner, T., \& Dolan, R. J. (2010). Experience and choice shape expected aversive outcomes. Journal of Neuroscience, 30, 9209-9215.

Shenhav, A., Straccia, M. A., Cohen, J. D., \& Botvinick, M. M. (2014). Anterior cingulate engagement in a foraging context reflects choice difficulty, not foraging value. Nature Neuroscience, 17, 1249-1254.

Simon, T. W. (1995). Democracy and social injustice: Law, politics, and philosophy. Rowman \& Littlefield.

Smith, D. V., Gseir, M., Speer, M. E., \& Delgado, M. R. (2016). Toward a cumulative science of functional integration: A meta-analysis of psychophysiological interactions. Human Brain Mapping, 37, 2904-2917.

Whitfield-Gabrieli, S., \& Nieto-Castanon, A. (2012). Conn: A functional connectivity toolbox for correlated and anticorrelated brain networks. Brain Connectivity, 2, 125-141.

Wicker, B., Keysers, C., Plailly, J., Royet, J. P., Gallese, V., \& Rizzolatti, G. (2003). Both of us disgusted in my insula: The common neural basis of seeing and feeling disgust. Neuron, $40,655-664$. 